\title{
Optimization of low sulfur carob pulp liquor as carbon source for fossil fuels biodesulfurization
}

\author{
Tiago P. Silva, Susana M. Paixão, Alexandra V. Teixeira, José C. Roseiro \\ and Luís Alves*
}

\begin{abstract}
BACKGROUND: Biodesulfurization (BDS) is a complementary technology to hydrodesulfurization since it allows the removal of recalcitrant sulfur compounds present in fossil fuels. The cost of culture medium to produce the biocatalysts is still one limitation for BDS application. Carob pulp, as an alternative carbon source, can reduce this cost. However, the presence of sulfates is critical, since BDS is inhibited at very low concentrations. Thus, the goal of this work was to optimize the process of sulfur precipitation on carob pulp liquor.
\end{abstract}

RESULT: The effect of $\mathrm{BaCl}_{2}$ concentration (0-0.5\%) and exposure time (6-36 h) on sulfate removal from carob pulp liquor was studied according to a statistical design following the Doehlert distribution for two factors. This experimental design determined that $0.5 \% \mathrm{BaCl}_{2}$ concentration for $21 \mathrm{~h}$ were adequate conditions for sulfate removal from carob pulp liquor using BDS.

CONCLUSION: These results demonstrate that it is possible to use alternative carbon sources derived from agro-industrial wastes for BDS, even those with high sulfur levels. For future industrial application, an inexpensive culture medium would have to be employed in a large-scale process and carob pulp liquor could be the carbon source.

(c) 2012 Society of Chemical Industry

Keywords: biodesulfurization; sulfate precipitation; Gordonia alkanivorans; carob pulp; experimental design

\section{INTRODUCTION}

The combustion of fossil fuel generates emissions of sulfur dioxide, nitrogen oxides, volatile organic compounds and particulate matter. These toxic compounds are responsible for environmental pollution and acute and chronic effects on human health. ${ }^{1}$ Also, the primary cause of acid rain is the washout of corrosive substances that are produced by the reaction of sulfur and nitrogen oxides with other chemicals present in the atmosphere. ${ }^{2}$

In recent years there has been increasing concern regarding the sulfur levels in fossil fuel and its derivatives. Excess sulfur might damage engine and exhaust after-treatment systems leading to higher air pollutant emissions. These emissions from road transport can be controlled through changes in fuel composition. To obtain the required levels of sulfur the most commonly used method is hydrodesulfurization (HDS), which uses extremely high temperatures and pressures, as well as expensive catalysts to bring the sulfur levels down. This method can remove mercaptans, thiophenes and benzothiophenes, but a large percentage of sulfur remains as recalcitrant organic compounds, such as dibenzothiophene (DBT) and its derivatives, ${ }^{3}$ which need even harsher conditions for their removal. These restrictions involve increased production costs and a loss in quality of the fuel.

Biodesulfurization (BDS) is a complementary technology to HDS. By using BDS it is possible to desulfurize most of the recalcitrant sulfur compounds under mild operating conditions using microorganisms, making it a simple and environment-friendly process. ${ }^{4}$ Several studies are being conducted using bacterial genera capable of performing BDS, which include Arthrobacter, Brevibacterium, Pseudomonas, Gordonia, and Rhodococcus. ${ }^{5}$ However, the BDS technique has some limitations, including the high costs of the culture medium, which makes the process very expensive. Thus, in order to reduce its costs, it is important to search for alternative carbon sources that can contribute to produce microbial biomass as well the necessary reducing equivalents required in BDS reactions.

Usually the use of agro-industrial residues is a sustainable strategy because of their low cost. ${ }^{6}$ The waste materials from the carob processing industry are among the industrial by-products most used as new carbon sources for several bioprocesses. ${ }^{7-9}$ Carob industrial wastes are small carob kibbles with a high content of soluble sugars, mainly sucrose, glucose, and fructose, which are easily extractable by water, producing sugar-rich syrups. ${ }^{10}$

However agro-residues usually contain large amounts of sulfates, which limits their application in BDS processes. The

\footnotetext{
Correspondence to: Luis Alves, LNEG - Bioenergy Unit, Estrada do Paf̧o do Lumiar, 22, 1649-038, Lisboa, Portugal.E-mail: luis.alves@ineg.pt

LNEG - Bioenergy Unit, Estrada do Paço do Lumiar, 22, 1649-038, Lisboa, Portugal
} 